\title{
Research of Intelligent Dispatching Operation Order Expert System
}

\author{
Miao Liu/ Yushen Jia/Qing Zhang \\ College of Mechanical and Electrical Engineering \\ Agricultural University of Hebei \\ Baoding, China \\ e-mail: 1379620953@qq.com \\ Corresponding author:Qing Zhang.
}

\author{
Qingjie Zhou/Xinghua Zhou \\ Beijing Join Bright Digital Power Technology Co., \\ LTD \\ Beijing, China \\ e-mail: 65471694@qq.com
}

\begin{abstract}
The intelligent dispatching operation order expert system is designed in order to improve the power grid dispatching automation level and reduce workload of the dispatchers. The concept of the equipment container model is applied to this system to establish knowledge-representation of power grid topology and knowledge-representation of dispatch operation rules, and research the reasoning technology of intelligent reasoning tree. Safety check based on static safety analysis is done for operation steps during the inferential process. It is proved in practice that the system has improved the feasibility of dispatching operation ticket and reduced the work intensity of the dispatchers.
\end{abstract}

Keywords-dispatching operation ticket; safety-checking; expert system

\section{INTRODUCTION}

Electric Power Dispatching Center is the core of electricity production and operation, the command center of electricity production and electricity distribution. As the conductor of the power grid, the dispatcher assumes the responsibility to let the network runs safely. Nowadays, the network's operation method is complex and changing; moreover, the devices often run at their extreme situation. The complexity of the switching operation causes the enormous workload for the dispatcher. To a large extent, the operation scheduling system reduced the workload of the dispatcher[1-5]. However, most of the operation scheduling systems has a low intelligence, and it only considered the constraint of the electrical connection relationship, without considering the static security constraints of the network. The result often bases on the experiment of the dispatcher, lacking of calculating and analyzing. In this case, developing an intelligent operation scheduling system has great significance. The operation scheduling system is mentioned in this article realized intelligent reasoning function, and is able to check the safety based on static security analysis, to make the operation safe and stable.

\section{SYSTEM OVERALL DESIGN}

The expert operation scheduling system software has a frame as Graph 1. The system realizes graphical interface, automatic operation, intelligent reasoning, safety checking, and system maintenance. Reasoning device does intelligent reasoning bases on the knowledge from the rule base and the real-time data from the SCADA system, and generates operation sequence by analyzing the purpose of the operation. It finds out the steps needed to be checked, and checks its safety with static safety analyzing to analyze the existence of safety problems, then ends the reasoning and generates correct operation ticket if it finds no problem.

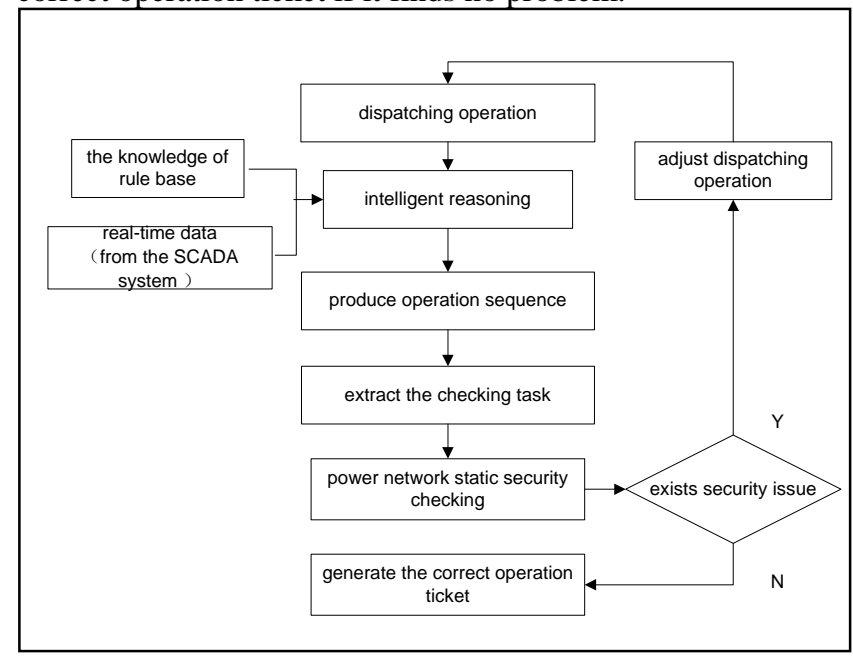

Figure 1. Framework of system software.

\section{KNOWLEDGE REPRESENTATION OF THE EXPERT SYSTEM}

The common information model of IEC 61970 presented a concept of device container model. Device container describes a method to organizing and naming a device: every container represents other containers or device objects' assemble. The maintain device containers includes plants, wiring type and interval. A device belongs to a device container, and the container probably be contained by other containers. For example, a switch belongs to an interval (container), and the interval perhaps belongs to a wiring type (device container), and the wiring type belongs to plant (container).This article applies the concept of device container model to the expert operation scheduling system, in order to increase the speed of topological analysis ,reasoning and the operation ticket making.

\section{A. Network Representation of Topology Knowledge}

The key to expert ability is the knowledge of it. The quality of an expert system largely depends on the how much and what level its knowledge have. The expert operation 
scheduling system's basis is knowledge of network topology; effective network structure knowledge is the premise of the success to an expert system. In order to facilitate applying the device container model, this article divides the devices into intervals, and researches the knowledge representation of the device interval mainly. Whether the line is charged is controlled by breaker, so the line is divided by breakers generally. With the relating knife switch and the line, the switch interval is formed. The other elements, just like the generators, the transformers, the injection, made up the element interval; abstract the bus bar and the line into the element interval. For example, a three volumes transformer's interval is composited by the transformer and its high/mid/low pressure side. Property Settings of transformer interval:

- Switch Interval Position (0:High Pressure Side; 1:Mid Pressure Side; 2:Low Pressure Side).

- $\quad$ Switch Interval Device Type (0:Switch; 1:Knife Switch; 2:Earthing Knife Switch; 3: Earthling Line).

- $\quad$ Switch Interval Device Name.

\section{B. The Knowledge Representation of Scheduling Operation Rules}

The operation rules are the core of the expert system. It acts in concert with the network's connection method, realtime electrical device state and second device protection to guide the formulation to the order of operations. The network dispatching is integrated and varied, so the operating method can neither be described by an exact mathematic model nor a switch controlling logic. A rational operation scheduling rule should be developed to increase the versatility and maintainability of the dispatch operation ticket expert system.

Switching operation is an operation which could let the network turn to the target state that is suit to the network[6]. In the reasoning process, the safety check based on static safety analysis is needed; so the network's safety constraint should be considered in the process. In order to ensure the correct control to the protection and the automatic device, a safety check is also needed to the protection and automatic device. The protection and automatic device is usually set at the breaker. It could embed the controlling rule of the secondary devices to the controlling rule of the primary devices to make sure the secondary devices have correct action when the primary devices act. The control device realizes the automatic ticket make with the reasoning device, so the rule model should be fit to the reasoning function. As a result, this article uses dynamic control rule model to create controlling rule model with classification and stratification, corresponding to the device control rules' various type. The controlling rule model can be divided into bus bar, line, switch, transformer and conductor. Every category could be divided into device type, device property, operation tasks, container type, related devices, related devices' initial state and related devices' final state. Explain stratification design as Graph 2 below.

Among the graph, operation tasks represent the change of the primary device's running state, which is same to the regular rules of the network dispatch; container type, represents the container which the primary device belongs to; it mainly contains interval, connection method and plants. Related devices represents the devices that is contained by the container which the primary device belongs to. Related devices' initial state and final state represents the related devices' state at start and the target state. Using this performance method makes the rule easy to change and explain; it increased the versatility and maintainability of the dispatch operation ticket expert system.

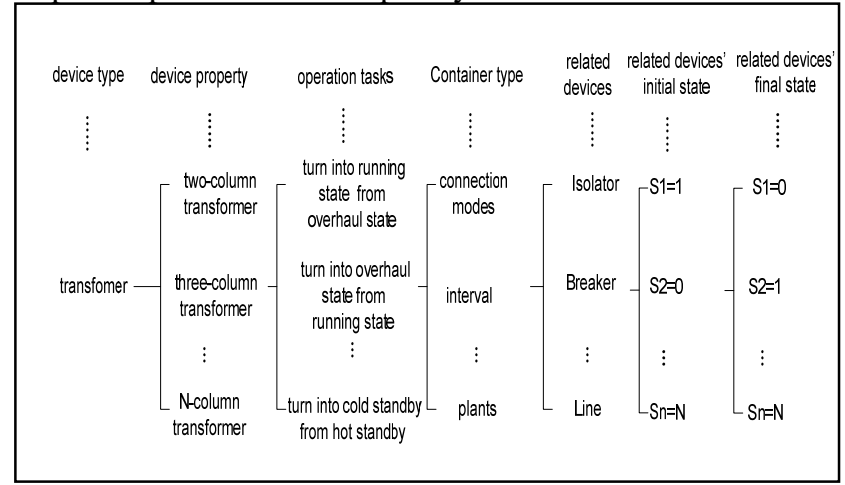

Figure 2. Operating rule of Transformer.

\section{The REASONING Method OF THE OpERATION TICKET SCHEDULING EXPERT SYSTEM}

\section{A. Intelligent Reasoning Principle}

Intelligent reasoning tree is a reasoning technology that has clear objectives, easy to expand, modify and achieve alternate with the users. The intelligent reasoning process is the process that the reasoning tree's growing and be trimmed. Generate a target node (target operating status) first when operating a device. A target node is corresponding to a line of operating command; then specific the operating to every breaking device, in other words, add several property nodes below the target node. One property node corresponds to a term wise command. Judge whether the property node needs to trigger other devices' operation in this interval container; do flow calculation after triggering to judge the static safety is satisfied. If not, find a new trigger node from the connection type containers out layer.

The tree's trimming equals the determination the reasoning plan. It could be divided into manual trimming and automatic trimming. Manual trimming is a manual screening process to the branches mainly; make rational dispatch operating ticket through the mutual with the dispatcher. Adopt the depth-first search strategy to Traverse and find the nodes needed interact and prompt the dispatcher to do branches selection. After a branch is selected, the system will show the result of choosing the node in graph, operating information, power-down information and tide information. If the dispatcher considers the branch have problem, the system provides return function and allows him to choose again. Traverse and find the next node needs interact. Automatic trimming is to list every rational plan. The user only needs to rehearse every plan and determine which to select at last. 


\section{B. Case Analysis}

Explain the reasoning and ticket-making process with a specific task. Graph 3 shows the connection diagram of a $35 \mathrm{KV}$ transformer substation. Switch 350 and Handcart Switch 600 is opened; other switches and knife switches are closed. The scheduling task is to let \#1 of the transformer substation turn into overhaul state from running state. The reasoning process is shown in Graph 4.

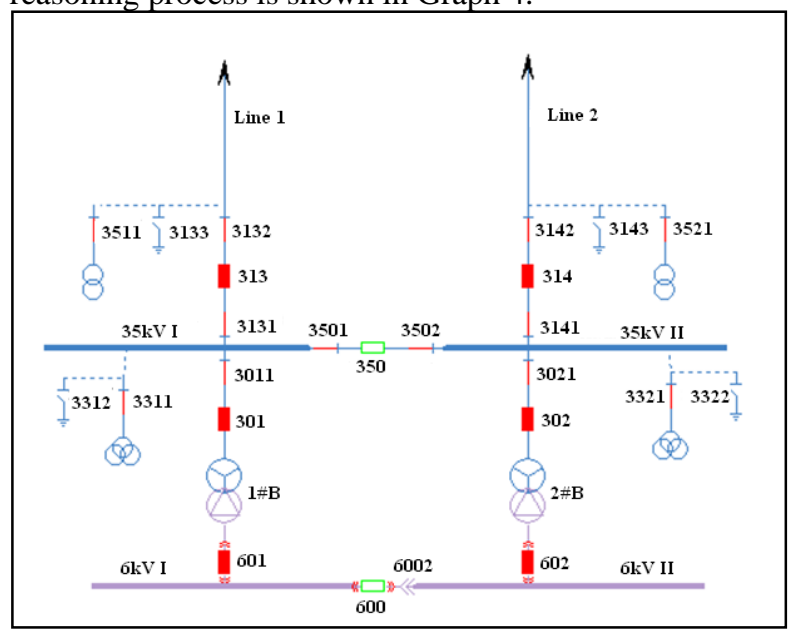

Figure 3. Substation wiring diagram.

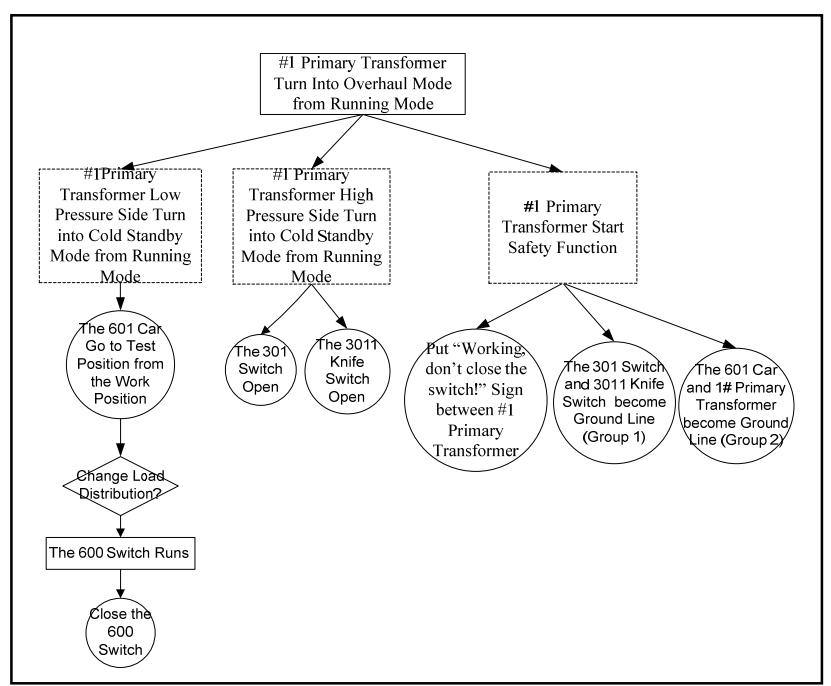

Figure 4. Reasoning process.

Produce target node "1\# Primary Transformer Turn into Overhaul from Running Mode”, add property node for the target node, then the reasoning device resolve the dispatch task and generate the specific operation sequence. The operation "601 Car Go to Test Position from Work Position" triggered the interactive node "whether to change load distribution", and the interactive node generate dispatch command and specific operation. Reasoning device find the task node - the solid line rectangular frame, in which the dispatch command are. Arrange them from underlying to upper layer; get the dispatch command "1: 600 Switch Start Running" and "2: $1 \#$ Primary Transformer Turn into Overhaul from Running Mode”.

\section{The Safety Check for Reasoning Process}

In order to increase the safety and the feasibility of the system, safety check based on static safety analysis is used through the reasoning process. The fundamental basis of network operation is network power flow, so static safety analysis is based on tide calculation. This article led static safety analysis constraint into reasoning device and provided reliable theoretical analysis for reasoning. Not all of the operations affect the safety of the network, so the safety check to all the operation steps is not needed. The system only needs to resolve the dispatch task and find the operations that need to check. Aiming to the found check task, the system uses PQ decomposition method for Fullflow calculation, and then does safety check just like cycle conditional judge, overload analysis and protection value analysis basing on the calculation result. Cycle conditional judge is to calculate the voltage difference between the ring points with static safety analysis function during the reasoning process, and judge whether the ring operation is feasible according to the dispatching procedure. Overload analysis is to judge whether active, reactive, current over limit and pressure over limit exists. Protection value analysis is to compare the tide current and the tide pressure at the breaker which has set up protection with the operating current and the operating pressure of the protection, and judging whether adjustment of the protection value is needed. The protection value should be adjusted if the tide value is bigger than the operating value.

\section{EPILOGUES}

This article led the conception of the device container model into dispatch schedule expert system, established the knowledge representation and the reasoning logic, and conducted safety check based on static safety analysis. The system has applied to the power system at Nan yang, Henan. Practice has proved that the system reduced the dispatchers' workload, increased the safety of making ticket and the safety-running managing level.

\section{REFERENCES}

[1] X. B. Zhang, H. B. Wang, X. C. Dong, Z. C. Wang, and P. Zhang, "Intelligent dispatching operation order expert system," Relay, vol. 34(20), pp. 32-35, 2006.

[2] P. Ding, Y. Zhang, F. L. Hou, and H. B. Wang, "Operation order generation system with self-check function for distribution network," Electric Power Automation Equipment, vol. 27(2), pp. 107-110, 2007.

[3] J. W. Gong, H. B. Sun, L. Tang, and B. M. Zhang, "Dispatching Order Generating Expert System Based on Network Analysis and Intelligent Reasoning," Power System Technology, vol. 30(17), pp. 19-24, 2006

[4] M. Zhou, J. W. Ren, G. Y. Li, et al, "A multi-agent based dispatching operation instructing system in electric power systems," Proceedings of the CSEE, vol. 24(4), pp. 58-62, 2004.

[5] C. X. Guo, C. B. Zhu, Y. J. Cao, and Z. X. Han, “An overall antimaloperation strategy based on multi-agent system and 
implementation of intelligent switching order generation system,” Power System Technology, vol. 30(4), pp. 50-54, 2006.

[6] Y. Wang and Y.H. Yang, "Development on regional dispatching order-sheet expert system with self-check function,” Power System Protection and Control, vol. 37(21), pp. 95-98, 2009.
[7] Q. Liu, M. Zhou, G. Y. Li, and B. Y. Guo, "Power System Dispatching Order Generating System with Calculation and Analysis Functions,” Power System Technology, vol. 29(7), pp. 68-73, 2005. 